\title{
Adapting pharmaceutical reimbursement policies to manage spending on high-priced drugs
}

\author{
Elizabeth Docteur ${ }^{1 *}$, Ruth Lopert ${ }^{2}$ \\ From 3rd International PPRI Conference 2015: Pharmaceutical Pricing and Reimbursement Policies: Challenges \\ Beyond the Financial Crisis \\ Vienna, Austria. 12-13 October 2015
}

\section{Problem statement}

Very high-priced pharmaceuticals also known as "specialty" drugs constitute a large and growing share of total spending on health care in OECD countries. Due to the lack of therapeutic alternatives and high perceived therapeutic value of the drugs, policies used to manage pharmaceutical spending are often impractical or ineffective in the case of specialty drugs. In this environment, there is a need to reformulate existing policies or develop new ones that are best suited to managing this growing group of products in a way that is consistent with the values and objectives underlying the policy frameworks of publicly financed health systems.

\section{Objective}

This study was undertaken to identify public policies being used internationally in the management of highpriced medicines and to evaluate their strengths and weaknesses in terms of managing costs while promoting affordable access to effective medicines.

\section{Methods}

The authors undertook reviews of the academic research and grey literature to identify policies used in OECD countries in the management of high-cost or specialty drugs, and information on the impact of the policies used on key outcomes. The review was supplemented by information from original interviews with pharmaceutical policy experts in selected countries. The authors compiled a list of policies being used and evidence on their impact, then undertook a policy analysis aimed at classifying the policies according to common features and implementation effects.

\footnotetext{
* Correspondence: edocteur@docteurconsulting.com

${ }^{1}$ Elizabeth Docteur Consulting, Alexandria, VA 22314, USA

Full list of author information is available at the end of the article
}

\section{Results}

Preliminary results from this ongoing study indicate that countries are employing a range of policy approaches in the management of high-priced pharmaceuticals, including price controls, delayed reimbursement decisions, application of utilization controls and implementation of cost sharing. However, in cases of nearly inelastic patient - as when a drug's therapeutic outcomes are perceived as very valuable with no significant market competition - specialty drug manufacturers succeed in obtaining effective sales prices at unprecedented levels. Alternative policy formulations, including those that shape the terms of the market in which buyers and sellers interact, may be necessary if policy-makers seek to change this paradigm.

\section{Conclusions}

Optimizing goals for management of high-cost pharmaceuticals may require trade-offs across them as informed by value judgments, meaning that there is likely no single best solution. Nevertheless, information on the strengths and weaknesses of alternative policies can be useful in efforts to choose appropriate policies in the face of uncertainty.

\section{Authors' details}

${ }^{1}$ Elizabeth Docteur Consulting, Alexandria, VA 22314, USA. ${ }^{2}$ George Washington University, Milken Institute of Public Health and Health Services, Department of Health Policy, 950 New Hampshire Ave, Washington, DC 20052, USA.

\section{Published: 5 October 2015}

\section{doi:10.1186/2052-3211-8-S1-O2}

Cite this article as: Docteur and Lopert: Adapting pharmaceutical reimbursement policies to manage spending on high-priced drugs. Journal of Pharmaceutical Policy and Practice 2015 8(Suppl 1):02.
() Biomed Central

(c) 2015 Docteur and Lopert This is an Open Access article distributed under the terms of the Creative Commons Attribution License (http://creativecommons.org/licenses/by/4.0), which permits unrestricted use, distribution, and reproduction in any medium, provided the original work is properly cited. The Creative Commons Public Domain Dedication waiver (http://creativecommons.org/ publicdomain/zero/1.0/) applies to the data made available in this article, unless otherwise stated. 